From the Departments of Ophthalmology and Pathology of the University of Michigan Hospital, Ann Arbor. Supported by Grant No. B-2873 of the U.S. Department of Health, Education, and Welfare

\title{
PORES IN THE INTERNAL LIMITING MEMBRANE OF THE HUMAN RETINA*)
}

BY

\author{
J. Reimer Wolter, M. D., Ann Arbor, Michigan
}

Pores can be demonstrated in the internal limiting membrane of the normal human retina. These pores are located along the branches of retinal blood vessels and allow for the migration of phagocytes between retina and vitreous space. In the periphery of the normal retina of eyes of virtually all persons over 40 years of age strands extend from the vitreous through the pores into the retina and surround blood vessels.

The internal limiting membrane of the human retina is a basement membrane formed and supported in a mosaic-like pattern by the inner ends of Müller's radial fibers. A definite area of thinning of the internal limiting membrane is known to exist along the larger branches of retinal blood vessels. This thinning is believed to be due to the separation of the inner ends of Müller's fibers caused by the blood vessels which results in the absence of these processes on top of retinal blood vessels. Wolff (1) stated that "the internal limiting membrane will be absent where a large vessel comes close to the hyaloid."

\section{HISTOLOGICAL OBSERVATIONS}

The normal retina of a well-fixed eye of a 68 -year-old white female patient is used in this paper to demonstrate the pores of the internal limiting membrane. Clinically, this eye exhibited an elevated brownish lesion in the macular area. The diagnosis of a central choroidal melanoma was made and the eye was enucleated. Histological examination, however, revealed the brown central

*) Received March 26th 1964. 
lesion to be hemorrhage and neovascularization between pigment epithelium and Bruch's membrane in the macular region. There was a break in Bruch's membrane. The pigment epithelium was detached, but exhibited normal cytology. The central retina appeared entirely normal. Thus, this is a case of the earliest stage of disciform macular degeneration in which the retina shows no involvement.

Serial sections of this eye show pore-like openings in the inner limiting membrane of the retina which are found next to retinal blood vessels. Fig. 1 shows such a pore (arrow) in the area of a crossing of a retinal artery and vein. It should be emphasized that serial sections next to the photographed region show the internal limiting membrane continuous over the blood vessels. This indicates that the defect seen in Fig. 1 represents a pore and not a more extensive slit-like defect in this membrane.

Fig. 2 gives an indication of the function of the pores in the internal limiting membrane. A phagocyte is seen just in front of the pore in the photograph. Such phagocytes are known to move under many conditions from the retina into the vitreous space. They actually are retinal microglia (2). Details in Fig. 3 are of special interest in this study. In this area a pore (arrow) is found on top of a rather small retinal vessel (v) that does not have very close contact with the inner retinal surface. Furthermore, the pore in this instance is located next to - and not within the area of thinning of the internal limiting membrane caused by the separation of the Müller fibers. The Müller fibers in the area of the pore in Fig. 3 are undisturbed in their arrangement by the blood vessel. Again, several phagocytes are seen in the vitreous next to the pore.

The pores in the inner limiting membrane are small. Earlier studies (2) of the migration of microglia from the retina into the vitreous in a case of intraocular tumor had already shown that microglia has to stretch to get through the pores. Figs. $4 \mathrm{a}$ and $\mathrm{b}$ show such microglia in two stages of their way through a pore. The internal limiting membrane is unstained in these photographs. However, the shape of the cells in the process of leaving the retina remind of a cat going through a small hole - and is indirect evidence of a narrow path.

The pores in the internal limiting membrane are smaller in the retinal periphery of the present case. However, they can be found - again usually located on blood vessels. It is most interesting to observe that vitreous strands commonly insert where the pores are (Fig. 5). Special stains show that such vitreous strands in the peripheral retina not only go to the area of these pores, but actually extend right through the pores to branch and surround retinal blood vessels in the area of the pores (Fig. 6).

The dense vitreous strands that extend to peripheral retinal blood vessels can be especially well demonstrated after trypsin digestion (3) of the retina. Fig. 7 shows an area of peripheral retina of an old normal human eye after trypsin digestion and silver stain (4). The internal limiting membrane is present, but 


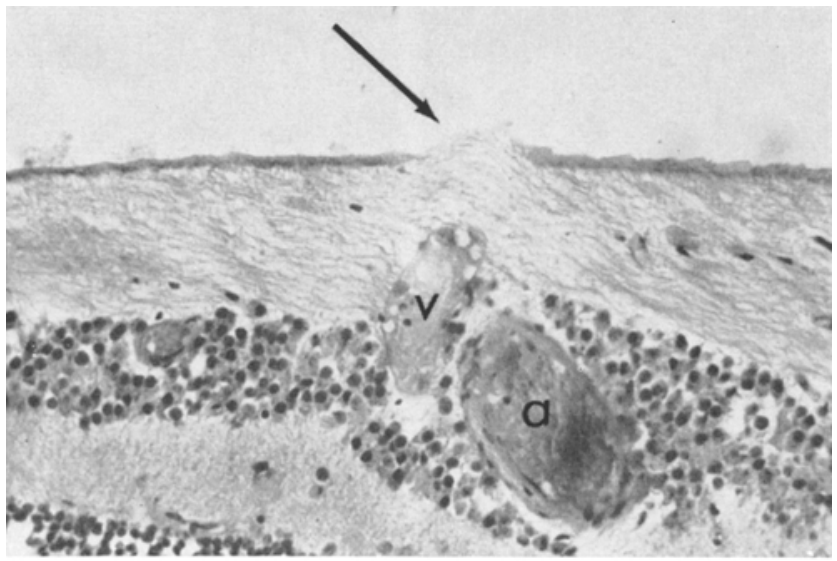

Fig. 1.

Pore (arrow) next to crossing artery and vein (a, v) in a normal retina with central hemorrhage under the pigment epithelium. - Paraffin section, $\mathrm{H}$ and $\mathrm{E}$ stain, photomicrograph $\times 300$.

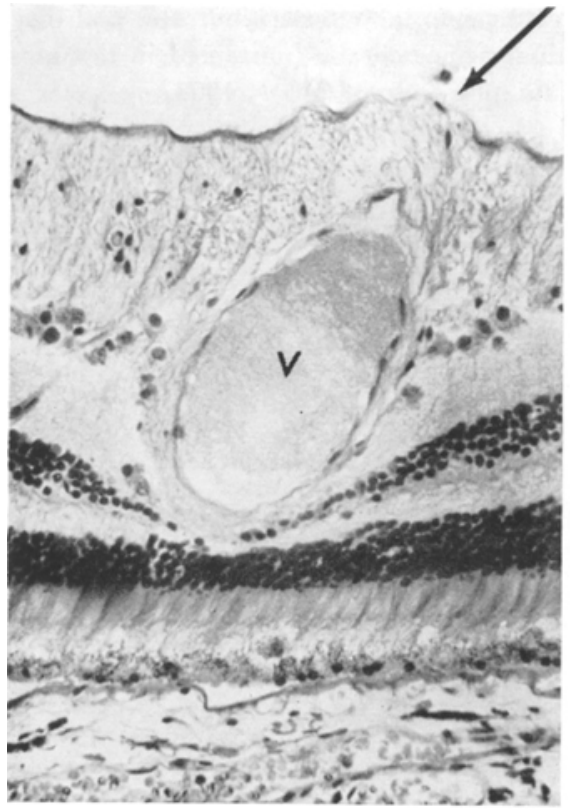

Fig. 2.

Small pore in the internal limiting membrane (arrow) of the same retina with a phagocyte next to it. Blood vessel: v. Paraffin section, $\mathrm{H}$ and $\mathrm{E}$ stain, photomicrograph $\times 300$.

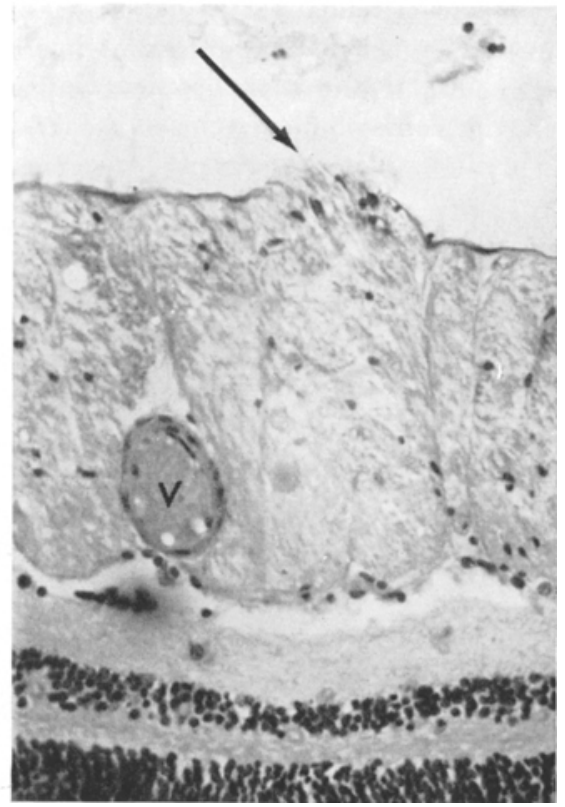

Fig. 3.

Pore in internal limiting membrane (arrow) with phagocytes next to it. Blood vessel: v. - Paraffin section, $H$ and $E$ stain, photomicrograph $\times 300$. 
lesion to be hemorrhage and neovascularization between pigment epithelium and Bruch's membrane in the macular region. There was a break in Bruch's membrane. The pigment epithelium was detached, but exhibited normal cytology. The central retina appeared entirely normal. Thus, this is a case of the earliest stage of disciform macular degeneration in which the retina shows no involvement.

Serial sections of this eye show pore-like openings in the inner limiting membrane of the retina which are found next to retinal blood vessels. Fig. 1 shows such a pore (arrow) in the area of a crossing of a retinal artery and vein. It should be emphasized that serial sections next to the photographed region show the internal limiting membrane continuous over the blood vessels. This indicates that the defect seen in Fig. 1 represents a pore and not a more extensive slit-like defect in this membrane.

Fig. 2 gives an indication of the function of the pores in the internal limiting membrane. A phagocyte is seen just in front of the pore in the photograph. Such phagocytes are known to move under many conditions from the retina into the vitreous space. They actually are retinal microglia (2). Details in Fig. 3 are of special interest in this study. In this area a pore (arrow) is found on top of a rather small retinal vessel $(v)$ that does not have very close contact with the inner retinal surface. Furthermore, the pore in this instance is located next to - and not within the area of thinning of the internal limiting membrane caused by the separation of the Müller fibers. The Müller fibers in the area of the pore in Fig. 3 are undisturbed in their arrangement by the blood vessel. Again, several phagocytes are seen in the vitreous next to the pore.

The pores in the inner limiting membrane are small. Earlier studies (2) of the migration of microglia from the retina into the vitreous in a case of intraocular tumor had already shown that microglia has to stretch to get through the pores. Figs. $4 \mathrm{a}$ and b show such microglia in two stages of their way through a pore. The internal limiting membrane is unstained in these photographs. However, the shape of the cells in the process of leaving the retina remind of a cat going through a small hole - and is indirect evidence of a narrow path.

The pores in the internal limiting membrane are smaller in the retinal periphery of the present case. However, they can be found - again usually located on blood vessels. It is most interesting to observe that vitreous strands commonly insert where the pores are (Fig. 5). Special stains show that such vitreous strands in the peripheral retina not only go to the area of these pores, but actually extend right through the pores to branch and surround retinal blood vessels in the area of the pores (Fig. 6).

The dense vitreous strands that extend to peripheral retinal blood vessels can be especially well demonstrated after trypsin digestion (3) of the retina. Fig. 7 shows an area of peripheral retina of an old normal human eye after trypsin digestion and silver stain (4). The internal limiting membrane is present, but 


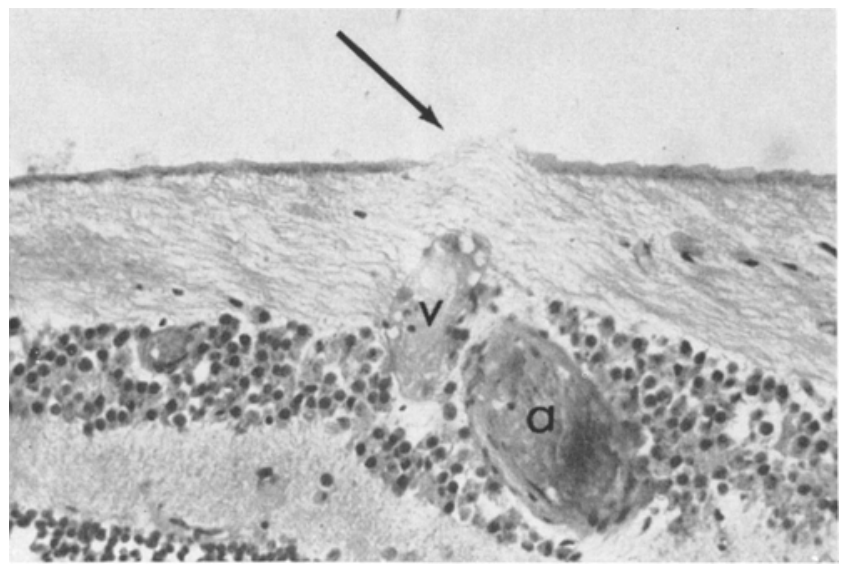

Fig. 1.

Pore (arrow) next to crossing artery and vein $(a, v)$ in a normal retina with central hemorrhage under the pigment epithelium. - Paraffin section, $\mathrm{H}$ and $\mathrm{E}$ stain, photomicrograph $\times 300$.

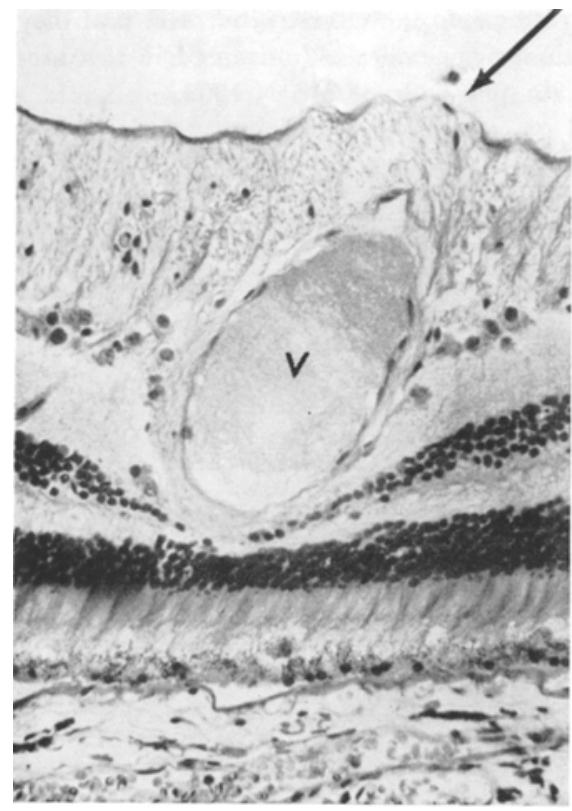

Fig. 2.

Small pore in the internal limiting membrane (arrow) of the same retina with a phagocyte next to it. Blood vessel: v. Paraffin section, $\mathrm{H}$ and $\mathrm{E}$ stain, photomicrograph $\times 300$.

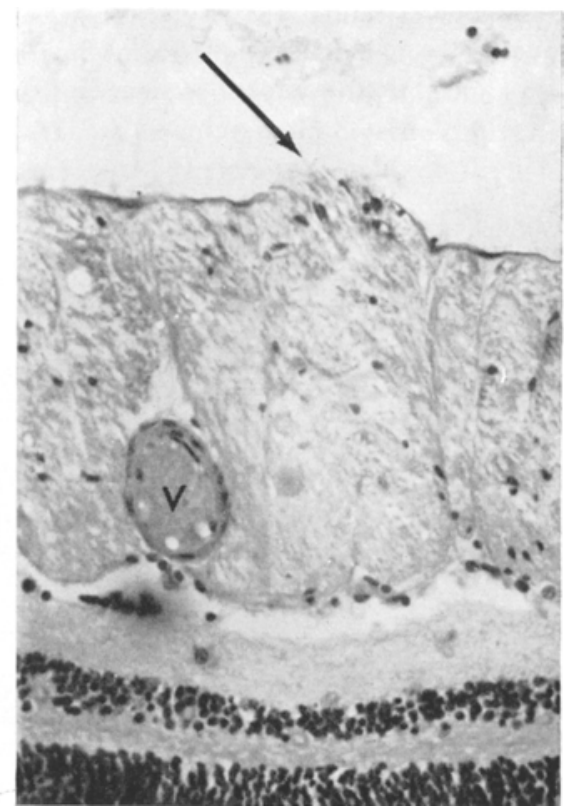

Fig. 3.

Pore in internal limiting membrane (arrow) with phagocytes next to it. Blood vessel: v. - Paraffin section, $H$ and $E$ stain, photomicrograph $\times 300$. 


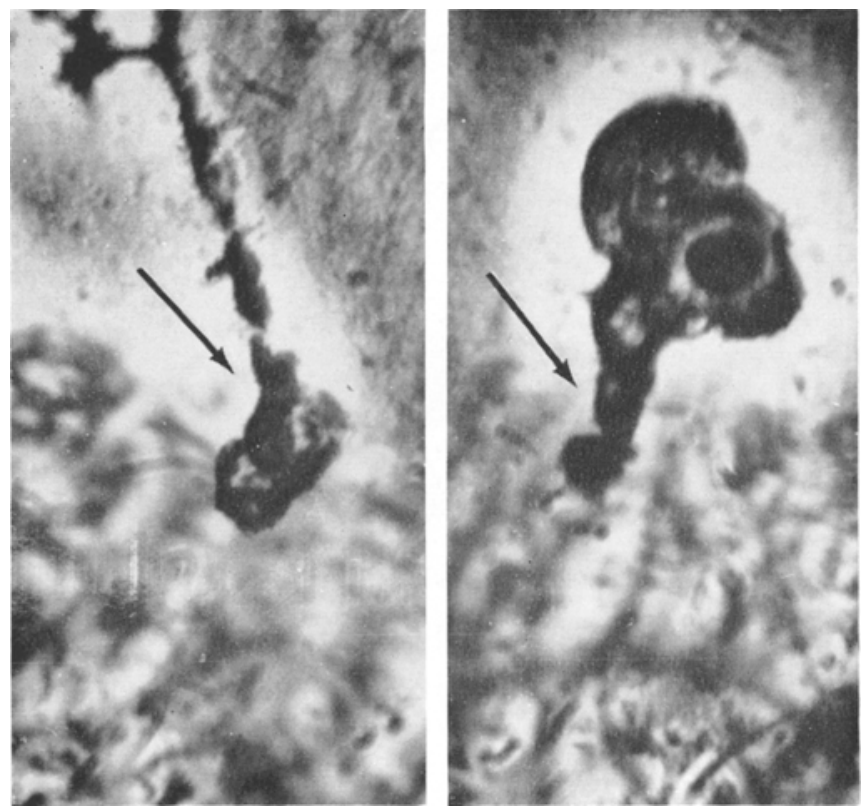

Figs. $4 a$ and $b$.

The shape of retinal phagocytes (arrows) migrating into the vitreous indicates that they squeeze through small pores in the internal limiting membrane (unstained in this section). - Frozen section, Hortega stain, photomicrograph $\times 1000$.

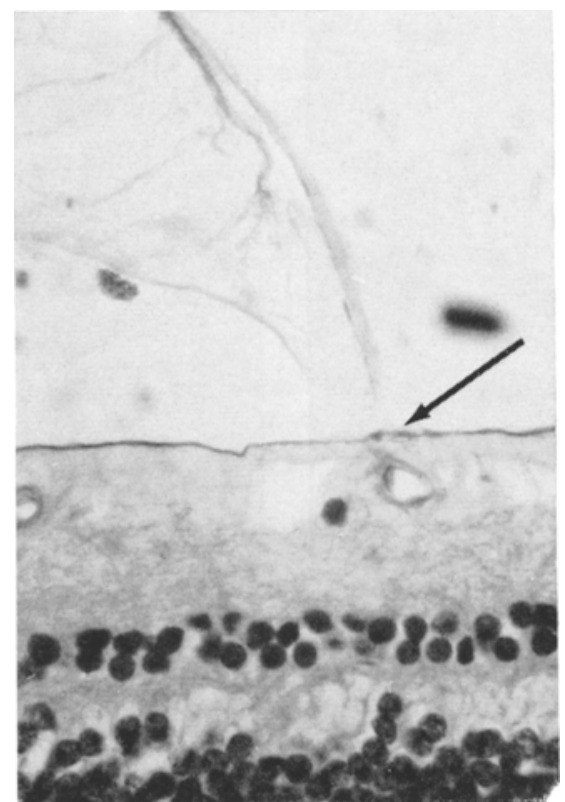

Fig. 5.

A vitreous strand (arrow) inserting in the region of a pore next to a blood vessel of the peripheral retina in the case of this paper. - Paraffin section, $H$ and $E$ stain, photomicrograph $\times 300$. 


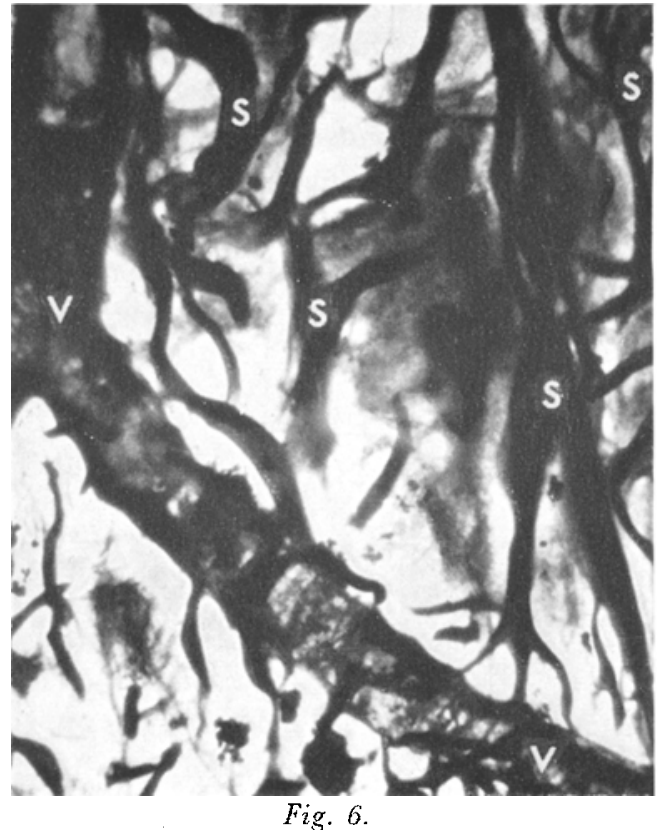

Preretinal vitreous strands (s) extending into the peripheral retina of a degenerated eyc and surrounding a blood vessel ( $v$ ) with finger-like processes. - Frozen section, Hortega stain, photomicrograph $\times 1000$.

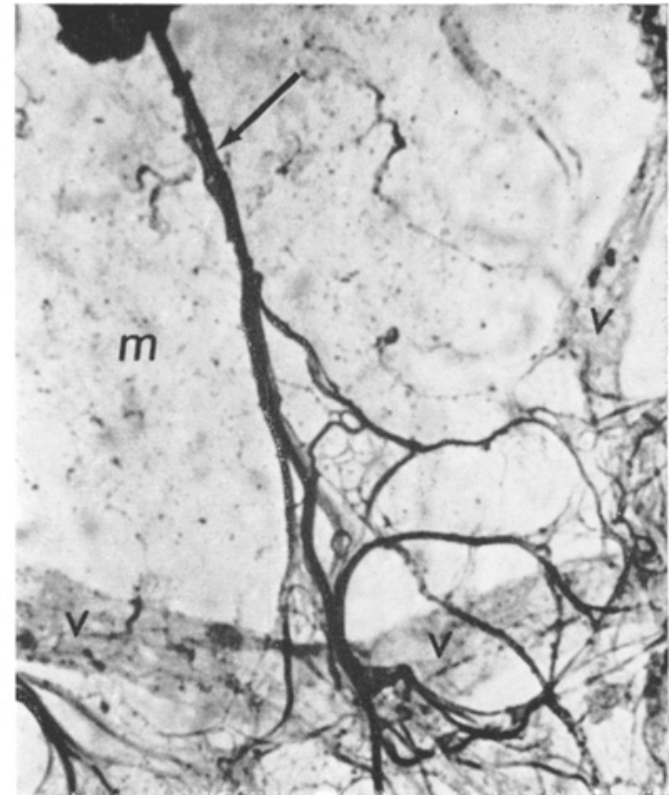

Fig. 7 .

A vitreous strand (arrow) extending through unstained internal limiting membrane $(m)$ to form a meshwork around blood vessels in the retina (v). -

Whole mount, trypsin digestion, silver stain, photomicrograph $\times 800$.

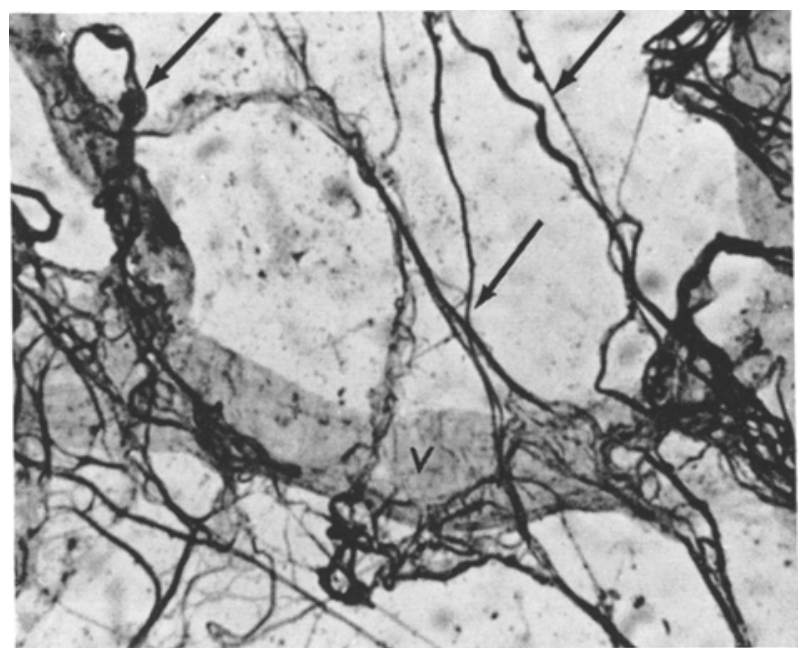

Fig. 8.

A group of vitreous strands (arrows) extending into the peripheral retina to form a meshwork of branching fibers around blood vessels $(v)$. - Whole mount, trypsin digestion, silver stain, photomicrograph $\times 800$. 


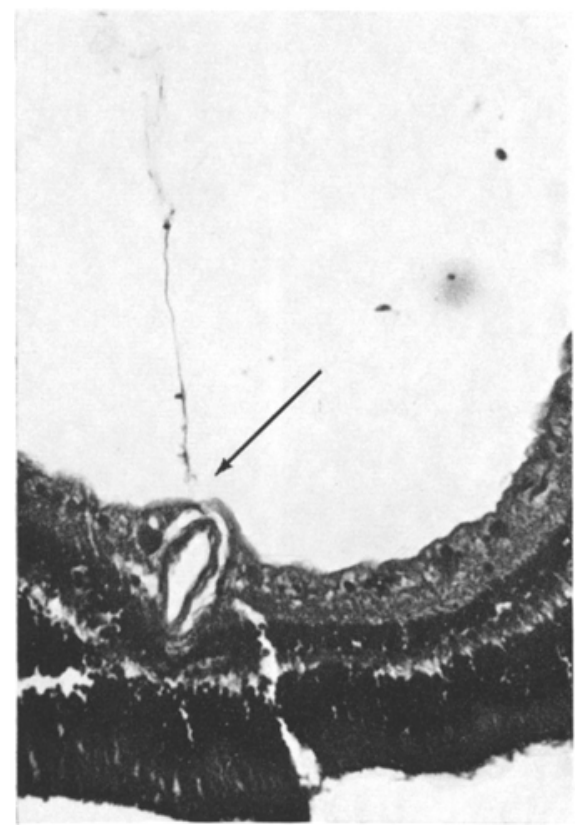

Fig. 9.

Dense vitreous strand inserting on a peripheral retinal blood vessel (arrow) in eye with absolute secondary glaucoma of 64-year-old patient. - Paraffin section, $\mathrm{H}$ and $\mathbf{E}$ stain, photomicrograph $\times 300$. 
unstained. A coarse vitreous strand (arrow) is seen to extend into the retina and form branches and a bizarre meshwork of fibers around retinal blood vessels (b). Several vitreous strands (arrows) come through pores into the peripheral retina and surround blood vessels (b) in a similar area photographed in Fig. 8. A vitreous strand inserting in a pore of the peripheral retina from an eye with more advanced degeneration is seen in Fig. 9. This eye had two peripheral horse-shoe holes with vitreous strands and fresh hemorrhage in front of one of these.

\section{DISGUSSION}

The observations of this study need little comment. The pores that are demonstrated here with routine stain in one case have actually been seen in many human eyes. Their number and size, however, vary a great deal in different individuals. One of the functions of these pores obviously is to let microglia migrate from the retina into the vitreous space. Our earlier study (2) indicates that the large microglia after extensive phagocytosis (gitter cells) cannot migrate from the vitreous back into the retina. They are often seen to pile-up in front of the retina - mainly next to blood vessels - and degenerate. We believe that the phagocytized substances that they contain may then reach the retinal blood vessels by diffusion. In less severe pathological involvement and in normal eyes, however, microglia probably moves freely back and forth between retina and vitreous.

It is hard to say at this stage what the relationship is between the pores in the peripheral retina and vitreous strands and whether the pores of the central retina and the pores containing strands in the peripheral retina are related formations. The fact remains, however, that vitreous strands of eyes with socalled degeneration of the anterior peripheral vitreous may be seen to enter the retina through small pores of the peripheral retina. The strands branch and surround blood vessels with a meshwork of fibers. It is well known that retinal holes with vitreous strands often occur in the area of a peripheral retinal blood vessel. This is the reason that retinal breaks commonly bleed. The observation that vitreous strands in such instances may actually be anchored on these blood vessels gives a new meaning to a clinically well known fact.

Mutlu and Leopold (5) recently demonstrated the same vitreous strands entering the peripheral retina and called them "vitreoretinovascular bands". It may be added here that it seems to me that the spider-like formations of degenerating perivascular glia seen in the peripheral retina (6) are distinctly different from the extensions of vitreous strands into the peripheral retina. Further studies should be aimed at the question whether the strands demonstrated in this and earlier studies (6) represent condensations of normal vitreous fibers or 
a new-formed element that develops with aging. Vitreous bands inserting on retinal blood vessels are, of course, not the only kind of vitreoretinal adhesions. The outer layer of the vitreous actually takes part in forming the normal internal limiting membrane of the retina. Persistence of this broad connection and strand-like degeneration within the vitreous body cause, for example, other types of vitreo-retinal bands.

Finally it has to be emphasized that the pores of the internal limiting membrane and the thinning of this membrane along the blood vessels are two different facts. Absence of the internal limiting membrane in larger areas along the blood vessels as described by Wolff (1) is not seen in our cases. However, the inner limiting membrane normally shows great variations in its thickness and the thinning along the larger retinal blood vessels may be quite outspoken - so that the membrane seems to be absent with some stains. Absence of the inner limiting membrane is normal, of course, on the optic disk (7).

\section{$S U M M A R Y$}

The internal limiting membrane of the human retina contains small pores that allow retinal microglia to migrate into the vitreous. Vitreous bands may extend through these pores peripherally and surround retinal blood vessels with their branches.

\section{REFERENCES}

1. Wolff, E.: The anatomy of the eye and orbit. The Blakiston Comp., Philadelphia and Toronto, 1948, p. 95.

2. Wolter, J. R.: The macrophages of the human vitreous body. Amer. J. Ophth. 49: $1185,1960$.

3. Kuwabara, T., and Cogan, D. G.: Studies of retinal vascular patterns. Arch. Ophth. 64: $904,1960$.

4. Wolter, J. R.: The pericytes of the human retina. Amer. J. Ophth. 53: 981, 1962.

5. Mutlu, F., and Leopold, $l$. H.: Structure of human retinal vascular system. Arch. Ophth. 71: 93, 1964.

6. Wolter, J. R., and Wilson, W. W.: Degeneration of the peripheral retina. Amer. J. Ophth. 47: 153, 1959.

7. Wolter, J. R.: Glia of the human retina. Amer. J. Ophth. 48: 370, 1959. 